\title{
Contextual interactions in a generalized energy model of complex cells
}

\author{
BABETTE K. DELLEN ${ }^{1,2, *}$, JOHN W. CLARK ${ }^{3}$ and RALF WESSEL ${ }^{3}$ \\ ${ }^{1}$ Bernstein Center for Computational Neuroscience Göttingen, Max-Planck-Institute for Dynamics \\ and Self-Organization, Bunsenstrasse 10, 37073 Göttingen, Germany \\ ${ }^{2}$ Intitut de Robòtica i Informàtica Industrial (CSIC-UPC), Llorens i Artigas, 08028 Barcelona, \\ Spain \\ ${ }^{3}$ Department of Physics, Washington University in Saint Louis, One Brookings Drive, St. Louis, \\ MO 63130, USA
}

Received 23 February 2007; accepted 11 April 2008

\begin{abstract}
We propose a generalized energy model of complex cells to describe modulatory contextual influences on the responses of neurons in the primary visual cortex (V1). Many orientationselective cells in V1 respond to contrast of orientation and motion of stimuli exciting the classical receptive field (CRF) and the non-CRF, or surround. In the proposed model, a central spatiotemporal filter, defining the CRF, is nonlinearly combined with a spatiotemporal filter extending into the nonCRF. These filters are assumed to describe simple-cell responses, while the nonlinear combination of their responses describes the responses of complex cells. This mathematical operation accounts for the inherent nonlinearity of complex cells, such as phase independence and frequency doubling, and for nonlinear interactions between stimuli in the CRF and surround of the cell, including sensitivity to feature contrast. If only the CRF of the generalized complex cell is stimulated by a drifting grating, the model reduces to the standard energy model. The theoretical predictions of the model are supported by computer simulations and compared with experimental data from V1.
\end{abstract}

\section{INTRODUCTION}

The responses of V1 neurons to a stimulus in the classical receptive field (CRF) are influenced by stimuli in the non-CRF or surround (Albright and Stoner, 2002). The responses represent not only information about stimulus features, but also about the context in which a stimulus is embedded. The contextual influences of neural responses correlate well with a variety of psychophysical phenomena, such as perceptual pop-out (Kastner et al., 1997; Knierim and Van Essen, 1992), contour

\footnotetext{
*To whom correspondence should be addressed. E-mail: bkdellen@bccn-goettingen.de
} 
integration (Kapadia et al., 1995), surface perception (MacEvoy et al., 1998; Rossi et al., 1996), and figure-ground segregation (Lamme, 1995; Lee et al., 1998; Zipser et al., 1996).

The majority of V1 neurons shows selectivity for the orientation, spatial frequency, and speed of a drifting grating within their CRF; many V1 neurons are also selective for the direction of motion (DeAngelis et al., 1993; Hubel and Wiesel, 1962; Movshon et al., 1978; Ringach, 2002, 2004). Two main classes of V1 neurons have been identified: simple and complex. Simple cells are sensitive to the phase of a drifting grating, while complex cells are not. Because of their linear properties, simple cells are commonly described by spatiotemporal filters followed by a halfwave rectification. Complex cells, however, do not behave linearly; their responses are either modelled by employing local spatial summation of simple-cell responses, or, alternatively, by adding the squared responses of two simple cells (Adelson and Bergen, 1985; Chance et al., 1999; Dellen et al., 2005; Hubel and Wiesel, 1962; Qian and Mikaelian, 2000; Skottun et al., 1991). The latter is known as the energy model of complex cells (Adelson and Bergen, 1985; Emerson et al., 1992). With its variations, it remains successful in predicting experimental data (Dellen et al., 2005; Rust et al., 2005; Touryan et al., 2005), but only as long as the surround of the neuron is not stimulated.

Further neurophysiological studies have revealed that V1 neuronal responses are far more complex than previously believed. It was shown that the response of a cell activated by a stimulus in its CRF is affected by stimuli in the surround (Cavanaugh et al., 2002; Grosof et al., 1993; Hegde and Felleman, 2003; Jones et al., 2001; Levitt and Lund, 1997; Li et al., 1999; Müller et al., 2003; Rossi et al., 2001; Sillito et al., 1995; Walker et al., 2000). These surround effects often represent information about 'feature' contrast of line orientation or direction of motion (Cavanaugh et al., 2002; Levitt and Lund, 1997). In particular, the response of many orientation-selective neurons in the primary visual cortex (V1) to a stimulus of optimal orientation in the CRF is often suppressed when the surrounding field is of similar orientation. In the complementary case when the surround is oriented orthogonal to the center stimulus, suppression is lifted; sometimes the response is even facilitated (Cavanaugh et al., 2002; Kastner et al., 1999; Lamme, 1995; Levitt and Lund, 1997; Sillito et al., 1995). Similarly, the response of V1 neurons to a moving stimulus is enhanced when the surround is moving in the opposite direction, compared to the case when the surround is moving coherently with the center stimulus (Cao and Schiller, 2003; Lamme, 1995; Orban et al., 1986).

Some neurons in the primary visual cortex display a property which is known as endstopping, i.e. they are selective for bars of specific lengths (Hubel and Wiesel, 1962). Experimental data suggest that endstopped neurons provide the neural basis for the computation of curvature (Dobbins et al., 1987), measuring the change of orientation, or orientation contrast, along a line. Endstopping might thus have its origin in a mechanism similar to that underlying feature contrast sensitivity. 
To date, it has remained unclear how surround areas should be incorporated into descriptive models of cortical neurons, partly because modulatory influences appear to be inherently nonlinear. Previously, we showed that energy computations performed by complex-cell models partially account for relative-motion sensitive effects (Dellen et al., 2005). This effect originates from inherent mathematical properties of energy computations. Importantly, the effect arises in non-directionally selective complex cells, which might account for the striking independence of featurecontrast sensitivity from feature sensitivity. However, due to limitations of the standard energy model, a treatment of center/surround interactions could not be given (Dellen et al., 2005). In this work, we include non-CRF areas by proposing a generalized model of complex cells in which two complex spatiotemporal filters of different spatial extent and spatiotemporal properties are combined nonlinearly. In this model, sensitivity to feature contrast, i.e. orientation and motion contrast, between the CRF and non-CRF arises from energy computations of complex cells. The fundamental and defining properties of complex cells, such as phase independence and frequency doubling, remain unaltered in the generalized description.

\section{RESULTS}

The generalized energy model of complex cells

In the energy model of complex cells (Adelson and Bergen, 1985) (Fig. 1A), the basic nonlinear response properties of V1 neurons are reproduced by multiplying a mathematically complex spatiotemporal filter with its complex conjugate,

$$
c^{\mathrm{AB}}(t)=s(t) s^{*}(t)
$$

where

$$
s(t)=\int\left[\int I\left(\mathbf{x}, t^{\prime}\right) W\left(\mathbf{x}, \mathbf{x}_{0}\right) \sum_{j} w^{j} \exp \left[i\left(\mathbf{k}^{j} \cdot \mathbf{x}-\psi^{j}\right)\right] \mathrm{d} \mathbf{x}\right] g\left(t-t^{\prime}\right) \mathrm{d} t^{\prime} .
$$

The function $W\left(\mathbf{x}, \mathbf{x}_{0}\right)=\left(1 / \pi \sigma^{2}\right) \exp \left[-\left(\mathbf{x}-\mathbf{x}_{0}\right)^{2} / \sigma^{2}\right]$ is a Gaussian of width $\sigma$ which defines the size of the CRF centered at $\mathbf{x}_{0}$. The wave vector $\mathbf{k}^{j}=$ $k\left(\cos \theta^{j}, \sin \theta^{j}\right)$ defines the spatial frequency $k$ and the spatial orientation $\theta^{j}$ of each spatial component of the model cell. The index $j$ identifies the orientation of the spatial component through $\theta^{j}=j \pi / 4$, where $j=0, \ldots, 7$. Each spatial component is multiplied by its weight $w^{j}$ and phase-shifted by $\psi^{j}$. The function $g(t)=t[\cos (\omega t)+i \cos (\omega t+\phi)] \exp (-t / \tau)$ is a complex temporal filter. The phase $\phi$ determines the directional selectivity of the filter. The functional form of the temporal filter resembles real temporal responses of V1 neurons (Chen et al., 2001; DeAngelis et al., 1993). The parameter $\tau$ determines the temporal decay of the response and $\omega$ is the angular frequency of the filter. Unless otherwise specified, the weights $w^{j}$ of the filter $s(t)$ are all zero except for $j=0$, where $\theta^{0}=0$. The integration is performed over the entire visual field. 
Motion-contrast sensitivity between stimuli within the CRF is an inherent property of complex cells, and it was shown that a straightforward extension of the standard model describes these types of nonlinear interactions in a satisfactory way (Dellen et al., 2005). The relative motion of a moving object and a moving background causes a characteristic oscillation of the complex-cell response in the standard energy model (Adelson and Bergen, 1985; Dellen et al., 2005). The oscillation frequency depends on the relative velocity of the moving entities. In the extended version (Dellen et al., 2005), we transformed this sensitivity into a change of the absolute amplitude by applying a linear temporal filter to the complex-cell response (see Fig. 1A). However, the standard energy model and its extended version provide a good approximation of actual complex-cell responses only as long as the non-CRF is not stimulated, but break down when the surround is stimulated simultaneously with the center. These nonlinear contextual interactions between center and surround are captured by neither model, simply because the area of integration of the spatial filters does not include the surround. To overcome this limitation, we augment the model with spatiotemporal filters that extend outside the CRF. These filters are not restricted to have the same spatiotemporal properties as the filter defining the center; instead, they can cover a broad range of spatial frequencies and orientations. Introducing all these spatiotemporal filters (or simple cells) results in a large spatiotemporal filter that integrates over the non-CRF:

$$
s_{\eta}(t)=\int\left[\int I\left(\mathbf{x}, t^{\prime}\right) W_{\eta}\left(\mathbf{x}, \mathbf{x}_{0}\right) \sum_{l} w_{\eta}^{l} \exp \left[i\left(\mathbf{k}_{\eta}^{l} \cdot \mathbf{x}-\psi_{\eta}^{l}\right)\right] \mathrm{d} \mathbf{x}\right] g_{\eta}\left(t-t^{\prime}\right) \mathrm{d} t^{\prime},
$$

where the index $\eta$ denotes the non-CRF. The function $W_{\eta}\left(\mathbf{x}, \mathbf{x}_{\mathbf{0}}\right)$ determines the size and shape of the non-CRF, and the wave vector $\mathbf{k}_{\eta}^{l}=k\left(\cos \theta_{\eta}^{l}\right.$, $\left.\sin \theta_{\eta}^{l}\right)$ defines the spatial frequency $k$ and the spatial orientation $\theta_{\eta}^{l}$ of each spatial component contributing to the non-CRF. The index $l$ specifies the orientation of the spatial component through $\theta_{\eta}^{l}=l \pi / 4$, where $l=0, \ldots, 7$. The spatial frequency of the non-CRF filter is taken to be identical to the spatial frequency of the CRF. Also, both the non-CRF and the CRF filter are centered at the same spatial position $\mathbf{x}_{0}$. Each spatial component is phase-shifted by $\psi_{\eta}^{l}$. The weights $w_{\eta}^{l}$ determine the spatial frequency selectivity and orientation selectivity of the filter. The function $g_{\eta}(t)=t\left[\cos \left(\omega_{\eta} t\right)+i \cos \left(\omega_{\eta} t+\phi_{\eta}\right)\right] \exp \left(-t / \tau_{\eta}\right)$ is a complex temporal filter, with the phase $\phi_{\eta}$ determining the directional selectivity of the filter. However, for simplicity, we chose the temporal filter of $s_{\eta}(t)$ not to be directionally selective, i.e. $\phi_{\eta}=0$. The standard energy model is generalized as follows. Instead of multiplying the filter $s(t)$ with its own complex conjugate (as in equation (1)), it is multiplied with the complex conjugate of the spatiotemporal filter $s_{\eta}(t)$ of the nonCRF, which extends beyond the boundaries of the CRF. The nonlinear response of the generalized model is then described by

$$
c(t)=s(t) s_{\eta}^{*}(t)
$$


As before, the multiplication operation ensures that the model complex cell only responds to stimuli that excite the CRF. However, the nonlinear operation has been adjusted to permit concurring stimuli present in the non-CRF to influence the cell's response, thereby fulfilling the defining properties of the non-CRF. In the last step of the algorithm, the response $c(t)$ is temporally filtered to increase sensitivity to feature contrast, and the absolute value of the real part of $c(t)$ is computed to ensure real-valued, positive responses (Dellen et al., 2005). In this final form of the generalized energy model, the complex-cells reads:

$$
\tilde{c}(t)=\left|\operatorname{Re}\left[\int s\left(t^{\prime \prime}\right) s_{\eta}^{*}\left(t^{\prime \prime}\right) h\left(t-t^{\prime \prime}\right) \mathrm{d} t^{\prime \prime}\right]\right|,
$$

with $h(t)=t \sin \left(2 \omega_{\mathrm{c}} t\right) \exp \left(-t / \tau_{\mathrm{c}}\right)$. A schematic of the generalized model is drawn in Fig. 1B. The weights $w_{\eta}^{l}$ will be selected to fit experimental data. To simplify the ensuing computations, we only use orientations $\theta_{\eta}^{l}$ ranging from 0 to $2 \pi$ in steps of $\pi / 4$.

\section{Computing relative direction of motion}

We investigate the response of the model to drifting sinusoidal gratings, which are widely used in experiment (Cavanaugh et al., 2002; DeAngelis et al., 1993; Hubel and Wiesel, 1962; Movshon et al., 1978; Ringach, 2002). The direction of drift is always orthogonal to the orientation of the grating. The CRF of the model complex cell is stimulated by a drifting grating $I\left(\mathbf{x}-\mathbf{v}_{\xi} \cdot t\right)$ of spatial frequency $\kappa=k$ and velocity $\mathbf{v}_{\xi}=v_{\xi}(1,0)$, which matches the preferred spatiotemporal frequency and orientation of the filter defining the center. For simplicity, we chose a complex cell that is not directionally selective and omit the imaginary part of the temporal filter. For the CRF filter, we compute:

$$
\begin{aligned}
& \operatorname{Re}[s(t)]=w^{0} \int\left[\int I\left(\mathbf{x}-\mathbf{v}_{\xi} \cdot t^{\prime}\right) W\left(\mathbf{x}, \mathbf{x}_{0}\right) \cos \left(\mathbf{k}^{0} \cdot \mathbf{x}-\psi^{0}\right) \mathrm{d} \mathbf{x}\right] \\
& \times \operatorname{Re}\left[g\left(t-t^{\prime}\right)\right] \mathrm{d} t^{\prime} \\
&=w^{0} \int\left[\int I(\mathbf{x}) W\left(\mathbf{x}+\mathbf{v}_{\xi} t^{\prime}, \mathbf{x}_{0}\right) \cos \left[\mathbf{k}^{0} \cdot\left(\mathbf{x}+\mathbf{v}_{\xi} t^{\prime}\right)-\psi^{0}\right] \mathrm{d} \mathbf{x}\right] \\
& \times \operatorname{Re}\left[g\left(t-t^{\prime}\right)\right] \mathrm{d} t^{\prime} \\
&=w^{0} \int {\left[A \cos \left(\mathbf{k}^{0} \cdot \mathbf{v}_{\xi} t^{\prime}-\psi^{0}\right)+B \sin \left(\mathbf{k}^{0} \cdot \mathbf{v}_{\xi} t^{\prime}-\psi^{0}\right)\right] } \\
& \times \operatorname{Re}\left[g\left(t-t^{\prime}\right)\right] \mathrm{d} t^{\prime} \\
&=w^{0} \int {\left[\sqrt{A^{2}+B^{2}} \cos \left[\mathbf{k}^{0} \cdot \mathbf{v}_{\xi} t^{\prime}-\psi^{0}-\arctan (B / A)\right]\right] } \\
& \times \operatorname{Re}\left[g\left(t-t^{\prime}\right)\right] \mathrm{d} t^{\prime} \\
& \approx w^{0} a \cos \left(k v_{\xi} t-\psi^{0}-\alpha\right),
\end{aligned}
$$




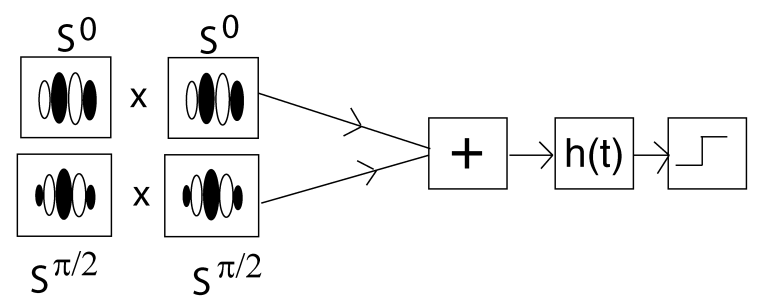

(A)

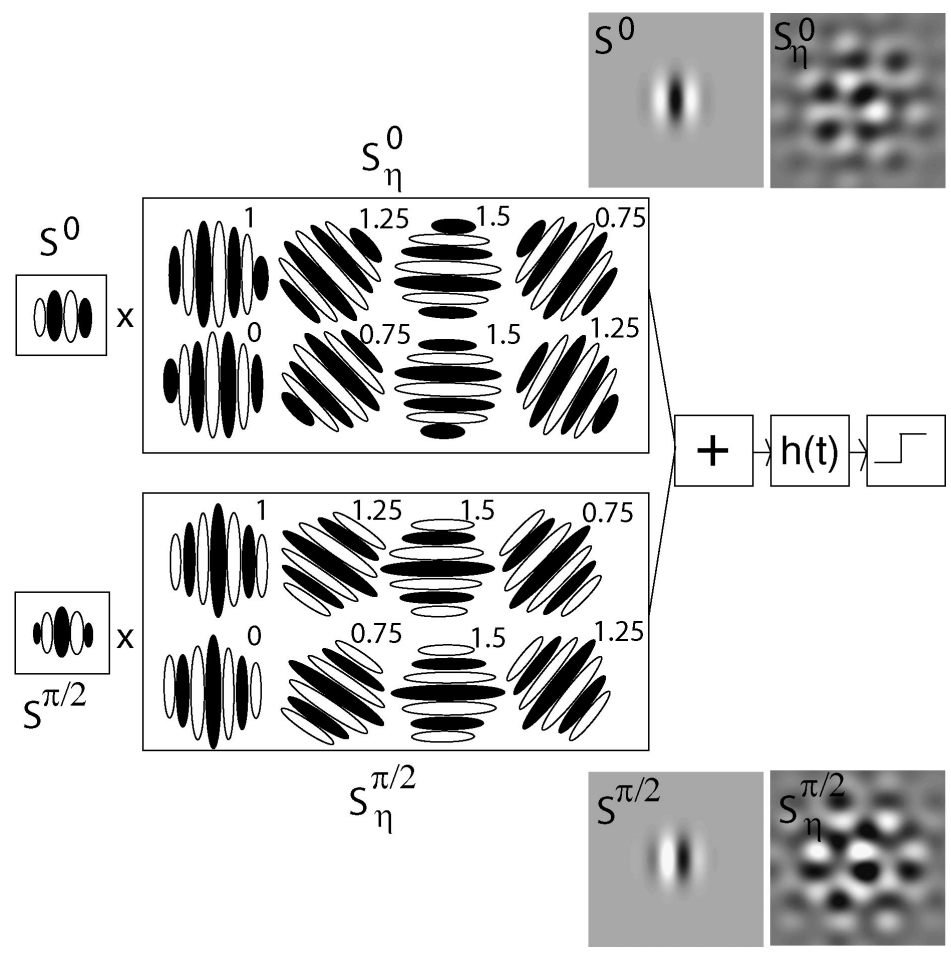

(B)

Figure 1. Schematics of energy models. (A) The extended standard energy model (Adelson and Bergen, 1985; Dellen et al., 2005). Taken together, two spatiotemporal filters $s^{0}$ and $s^{\pi / 2}$, differing in phase by $\pi / 2$, define a mathematically complex spatiotemporal filter that processes the input. The spatial components of the two filters are depicted in the upper and lower squares on the left. The filtered responses are multiplied with their complex conjugates (duplicated squares) and summed, returning the complex-cell response of the standard energy model (central square with plus sign). In the extended energy model, the complex-cell response is temporally filtered and rectified afterwards (squares on the right). This additional step generates amplitude tuning to relative motion. (B) In the generalized energy model of complex cells, the responses of a pair of spatiotemporal filters $s^{0}$ and $s^{\pi / 2}$ identical to those used in the standard model (see above) are multiplied by the complex conjugates of the responses obtained from a different pair of spatiotemporal filters, $s_{\eta}^{0}$ and $s_{\eta}^{\pi / 2}$, obtained via superposition of spatiotemporal filters extending into the non-CRF having different orientations (large rectangles) and carrying weights $w_{l}=(1,1.25,1.5,0.75,0,0.75,1.5,1.25)$ (see equation (35)). The spatial filters, as they are used in simulations, are presented as insets. The resulting values are summed, temporally filtered, and rectified. 
with

$$
\begin{aligned}
& A=\int I(\mathbf{x}) W\left(\mathbf{x}+\mathbf{v}_{\xi} \cdot t, \mathbf{x}_{0}\right) \cos \left(\mathbf{k}^{0} \cdot \mathbf{x}\right) \mathrm{d} \mathbf{x}, \\
& B=\int I(\mathbf{x}) W\left(\mathbf{x}+\mathbf{v}_{\xi} \cdot t, \mathbf{x}_{0}\right) \sin \left(\mathbf{k}^{0} \cdot \mathbf{x}\right) \mathrm{d} \mathbf{x},
\end{aligned}
$$

and

$$
\begin{aligned}
& a=\gamma \sqrt{A^{2}+B^{2}}, \\
& \alpha=\arctan (B / A),
\end{aligned}
$$

where $\gamma$ is a scaling factor that arises from the amplitude modulation by the temporal filter.

In the same way, we find

$$
\begin{aligned}
\operatorname{Im}[s(t)]= & \int\left[\int I\left(\mathbf{x}-\mathbf{v}_{\xi} \cdot t^{\prime}\right) W\left(\mathbf{x}, \mathbf{x}_{0}\right) \sin \left(\mathbf{k} \cdot \mathbf{x}-\psi^{0}\right) \mathrm{d} \mathbf{x}\right] \\
& \times \operatorname{Re}\left[g\left(t-t^{\prime}\right)\right] \mathrm{d} t^{\prime} \\
\approx & w^{0} a \sin \left(k v_{\xi} t-\psi^{0}-\alpha\right) .
\end{aligned}
$$

Hence, we have

$$
s(t) \approx w^{0} a \exp \left[i\left(k v_{\xi} t-\psi^{0}-\alpha\right)\right] .
$$

For the non-CRF filter, only the spatial components that concur with the orientation of the center grating contribute to the response. Hence, we obtain:

$$
s_{\eta}(t) \approx w_{\eta}^{0} a_{\eta} \exp \left[i\left(k v_{\xi} t-\psi_{\eta}^{0}-\alpha_{\eta}\right)\right]
$$

with

$$
\begin{aligned}
& A_{\eta}=\int I(\mathbf{x}) W_{\eta}\left(\mathbf{x}+\mathbf{v}_{\xi} \cdot t, \mathbf{x}_{0}\right) \cos \left(\mathbf{k}_{\eta}^{0} \cdot \mathbf{x}\right) \mathrm{d} \mathbf{x} \\
& B_{\eta}=\int I(\mathbf{x}) W_{\eta}\left(\mathbf{x}+\mathbf{v}_{\xi} \cdot t, \mathbf{x}_{0}\right) \sin \left(\mathbf{k}_{\eta}^{0} \cdot \mathbf{x}\right) \mathrm{d} \mathbf{x}
\end{aligned}
$$

and

$$
\begin{gathered}
a_{\eta}=\gamma_{\eta} \sqrt{A_{\eta}^{2}+B_{\eta}^{2}}, \\
\alpha_{\eta}=\arctan \left(B_{\eta} / A_{\eta}\right),
\end{gathered}
$$

where $\gamma_{\eta}$ is a scaling factor that arises from the amplitude modulation by the temporal filter. The complex-cell response (before applying the temporal filter) in the generalized model may then be written as:

$$
\begin{aligned}
c(t) & =s(t) s_{\eta}^{*}(t) \\
& =w^{0} w_{\eta}^{0} a a_{\eta} \exp \left[i\left(\psi_{\eta}^{0}-\psi^{0}+\alpha_{\eta}-\alpha\right)\right],
\end{aligned}
$$


where the trigonometric relationship $\cos (x) \cos (y)+\sin (x) \sin (y)=\cos (x-y)$ has been used in the last step. Note that the response of the model complex cell to the drifting grating is independent of time, which is also predicted by the standard energy model (Adelson and Bergen, 1985). Qualitatively, the generalized energy model reduces to the standard model provided only the CRF is stimulated with a drifting grating, although not quantitatively, since even in the center $W \neq W_{\eta}$.

Suppose, however, a surround grating of velocity $\mathbf{v}_{\mathrm{s}}=v_{\mathrm{s}}\left(\cos \theta_{\mathrm{s}}^{m}, \sin \theta_{\mathrm{s}}^{m}\right)$ having $v_{\mathrm{s}}=v_{\xi}$, orientation $\theta_{\mathrm{s}}^{m}=m \pi / 4$, and spatial frequency $k_{\mathrm{s}}=\kappa=k$, is added to the center stimulus. Then only the spatial components that concur with the orientation of either the surround- or center grating survive, and the response of the spatial filter in the non-CRF reduces to

$$
\begin{aligned}
s_{\eta}(t)= & w_{\eta}^{0} a_{\eta} \exp \left[i\left(k v_{\xi} t-\psi_{\eta}^{0}-\alpha_{\eta}\right)\right] \\
& +b_{\eta} w_{\eta}^{m+4} \exp \left[i\left(\mathbf{k}_{\eta}^{m+4} \cdot \mathbf{v}_{\mathrm{s}} t-\psi_{\eta}^{m+4}-\beta_{\eta}^{m+4}\right)\right] \\
& +b_{\eta} w_{\eta}^{m} \exp \left[i\left(\mathbf{k}_{\eta}^{m} \cdot \mathbf{v}_{\mathrm{s}} t-\psi_{\eta}^{m}-\beta_{\eta}^{m}\right)\right],
\end{aligned}
$$

with

$$
\begin{aligned}
& A_{\eta}^{l}=\int I(\mathbf{x}) W_{\eta}\left(\mathbf{x}+\mathbf{v}_{\xi} \cdot t, \mathbf{x}_{0}\right) \cos \left(\mathbf{k}_{\eta}^{l} \cdot \mathbf{x}\right) \mathrm{d} \mathbf{x}, \\
& B_{\eta}^{l}=\int I(\mathbf{x}) W_{\eta}\left(\mathbf{x}+\mathbf{v}_{\xi} \cdot t, \mathbf{x}_{0}\right) \sin \left(\mathbf{k}_{\eta}^{l} \cdot \mathbf{x}\right) \mathrm{d} \mathbf{x},
\end{aligned}
$$

and

$$
\begin{gathered}
b_{\eta}^{l}=\gamma_{\eta}^{l} \sqrt{\left(A_{\eta}^{l}\right)^{2}+\left(B_{\eta}^{l}\right)^{2}}, \\
\beta_{\eta}^{l}=\arctan \left(B_{\eta}^{l} / A_{\eta}^{l}\right) .
\end{gathered}
$$

Thus, for the complex-cell response we find

$$
\begin{aligned}
c(t)= & s(t) s_{\eta}^{*}(t) \\
= & w^{0} w_{\eta}^{0} a a_{\eta} \exp \left[i\left(\psi_{\eta}^{0}-\psi+\alpha_{\eta}-\alpha\right)\right] \\
& +w^{0} a b_{\eta}\left\{w_{\eta}^{m+4} \exp \left[i\left(\left(\mathbf{k}^{0} \cdot \mathbf{v}_{\xi}-\mathbf{k}_{\eta}^{m+4} \cdot \mathbf{v}_{\mathrm{s}}\right) t-\psi+\psi_{\eta}^{m+4}-\alpha+\beta_{\eta}^{m+4}\right)\right]\right. \\
& \left.+w_{\eta}^{m} \exp \left[i\left(\left(\mathbf{k}^{0} \cdot \mathbf{v}_{\xi}-\mathbf{k}_{\eta}^{m} \cdot \mathbf{v}_{\mathrm{s}}\right) t+\psi_{\eta}^{m}-\psi-\alpha+\beta_{\eta}^{m}\right)\right]\right\}
\end{aligned}
$$

where the trigonometric relationship $\exp (i x) \exp (i y)=\exp [i(x+y)]$ has been used.

Finally, let us construct the complex-cell response for different directions of motions of the surround grating. For $m=0$, we have

$$
\begin{aligned}
c(t)= & w^{0} w_{\eta}^{0} a a_{\eta} \exp \left[i\left(\psi_{\eta}^{0}-\psi+\alpha_{\eta}-\alpha\right)\right] \\
& +w^{0} a b_{\eta}\left\{w_{\eta}^{4} \exp \left[i\left(2 k v_{\xi} t+\psi_{\eta}^{4}-\psi-\alpha+\beta_{\eta}^{4}\right)\right]\right. \\
& \left.+w_{\eta}^{0} \exp \left[i\left(\psi_{\eta}^{0}-\psi-\alpha+\beta_{\eta}^{0}\right)\right]\right\},
\end{aligned}
$$


where only the contribution of the spatial component $l=4$ is time-dependent. Thus, subsequent temporal filtering with a bandpass filter of preferred temporal frequency $\omega=2 k v_{\xi}$ eliminates the time-independent terms, such that

$$
\tilde{c}(t) \approx \mid \operatorname{Re}\left\{w^{0} a b_{\eta}\left\{w_{\eta}^{4} \exp \left[i\left(2 k v_{\xi} t+\psi_{\eta}^{4}-\psi-\alpha+\beta_{\eta}^{4}\right)\right]\right\} \mid .\right.
$$

The temporally averaged response (denoted by \langle\rangle ) of the model complex cell is found to behave as:

$$
\langle\tilde{c}(t)\rangle \propto w^{0} w_{\eta}^{4} .
$$

In the same way, for the remaining surround-stimulus directions $\theta_{\mathrm{s}}^{m}$ we find

$$
\langle\tilde{c}(t)\rangle \propto w^{0} w_{\eta}^{m+4},
$$

where $m=0$ is identical to $m=8$. By adjusting the weights $w_{\eta}^{l}$ of the non-CRF filter, we are able to fit the tuning curve of the model complex cell to experimental data.

\section{Simulations}

Tuning to relative direction of motion. We have simulated the response of a directionally-selective generalized complex cell to drifting sinusoidal gratings. For the model complex cell, we choose the parameter values $k=1 \mathrm{cycle} / \mathrm{deg}$, $\sigma=0.6 \mathrm{deg}, \omega=4$ cycles $/ \mathrm{s}, \omega_{\eta}=4$ cycles $/ \mathrm{s}, \omega_{c}=8$ cycles $/ \mathrm{s}, \tau=0.088 \mathrm{~s}$, $\tau_{\eta}=0.088 \mathrm{~s}, \tau_{c}=0.05 \mathrm{~s}, \phi=-\pi / 2, \phi_{\eta}=0$, and $v_{\xi}=4 \mathrm{deg} / \mathrm{s}$. Unless otherwise specified, $w^{j}=1$ if $j=0$ and $w^{j}=0$ if $j \neq 0$. The parameter values are chosen such that the response of the model complex cell to a grating stimulus confined to the CRF reproduce measured V1 neuronal responses (Cavanaugh et al., 2002; DeAngelis et al., 1993; Hubel and Wiesel, 1962; Movshon et al., 1978; Ringach, 2002).

Experimentally, the properties of the non-CRF are less accessible than the CRF properties, due to the interaction between the non-CRF and the CRF. However, nonCRF influences on the CRF responses of V1 neurons suggest that the non-CRF is at least twice as large as the CRF (Cavanaugh et al., 2002). Accordingly, we choose a window function for the non-CRF to be three times as large as the size of the CRF, as illustrated in Fig. 1B. The weights $w_{\eta}^{l}=(1,1.25,1.5,0.75,0,0.75,1.5,1.25)$ with $l=(0,1,2, \ldots, 7)$, respectively, of the non-CRF filter are chosen based on the theoretically derived tuning curve of the surround interaction (see equation (35)), such that the model's tuning curves fit real tuning curves of V1 neurons with respect to different orientations/directions (Cavanaugh et al., 2002) of surround gratings. The phases $\psi_{\eta}$ of the spatial filters of the surround are chosen randomly, since their precise values have no influence on surround tuning properties of the model, as shown by theoretical analysis (equation (35)). The parameters are the same in all the simulations unless indicated otherwise.

Stimuli presented to the receptive field center are confined to a circular region whose diameter $d_{i}=1$ deg equals the spatial extent of the classical receptive field 
of the model cell; stimuli presented to the surround are confined to an annular region whose inner diameter is equal to the diameter of the center stimulus (Fig. 2, insets). The outer diameter of the annulus is $d_{0}=2 \mathrm{deg}$.

We first compute the response of the model complex cell to the center stimulus alone. The direction of motion of the center grating is varied from -180 to $180 \mathrm{deg}$ in steps of $45 \mathrm{deg}$. The responses are then integrated over a time course of $t=2.5 \mathrm{~s}$ and averaged over ten trials with refreshed gratings having randomly chosen phases. The results are plotted in Fig. 2A (black dots). The shape of the tuning curve reflects the selectivity of the model complex cell for direction. The tuning curve of an actual complex cell from monkey primary visual cortex to the same stimulus is plotted with the simulation data (Fig. 2A, open circles) (Cavanaugh et al., 2002). The model results are scaled to fit the experimental values, which are plotted just as presented in the original paper. Next, a drifting grating was added to the surround while keeping the direction of the center grating fixed at the cell's optimal direction and vary the direction of the surround grating from $-180 \mathrm{deg}$ to $180 \mathrm{deg}$ in steps of $45 \mathrm{deg}$. The results are plotted as black dots in Fig. 2B. The complex cell is tuned to the relative direction of center and surround, with lowest responses for coincident direction of motion and largest responses to gratings with orthogonal direction of motion. The response profile of the model complex cell resembles experimentally derived tuning curves from monkey primary visual cortex (Cavanaugh et al., 2002). Some experimental data are plotted for comparison (Fig. 2B, open circles). If the direction of the center grating is at $45 \mathrm{deg}$ instead of at the optimal direction of the model complex cell, the tuning curve flattens (Fig. 2C, black dots), a phenomenon that has been observed in primary visual cortex (Cavanaugh et al., 2002) (Fig. 2C, open circles). The model results presented in Figs. 2B and 2C are scaled and shifted to fit the experimental values, which are plotted as presented in the original paper. Since the model proposed here is an entirely functional description of cortical complex cells, we set the concrete neuronal implementation of this normalization aside. However, in a biophysical model, the normalization could be modelled by incorporating a uniform, isotropic surround suppression (see Discussion).

\section{Influence of center stimulus attributes on orientation-contrast sensitivity}

We have investigate the influence of center contrast on orientation contrast sensitivity of the model complex cell. The center stimulus is a grating of the model complex cell's preferred orientation and spatial frequency. The surround stimulus is a grating of the model complex cell's preferred spatial frequency. We compute the response as a function of the center contrast for different surround stimulus orientations. In Fig. 3A, the results for different surround stimulus orientations of 90, 45, and 0 deg are plotted as dashed black, solid black, and solid gray lines, respectively. The strength of the model complex cell's sensitivity to orientation contrast depends on the contrast of the center stimulus, which is a consequence of the nonlinear computations of the complex cell model. Similar effects have been observed in real V1 responses (Cavanaugh et al., 2002). 

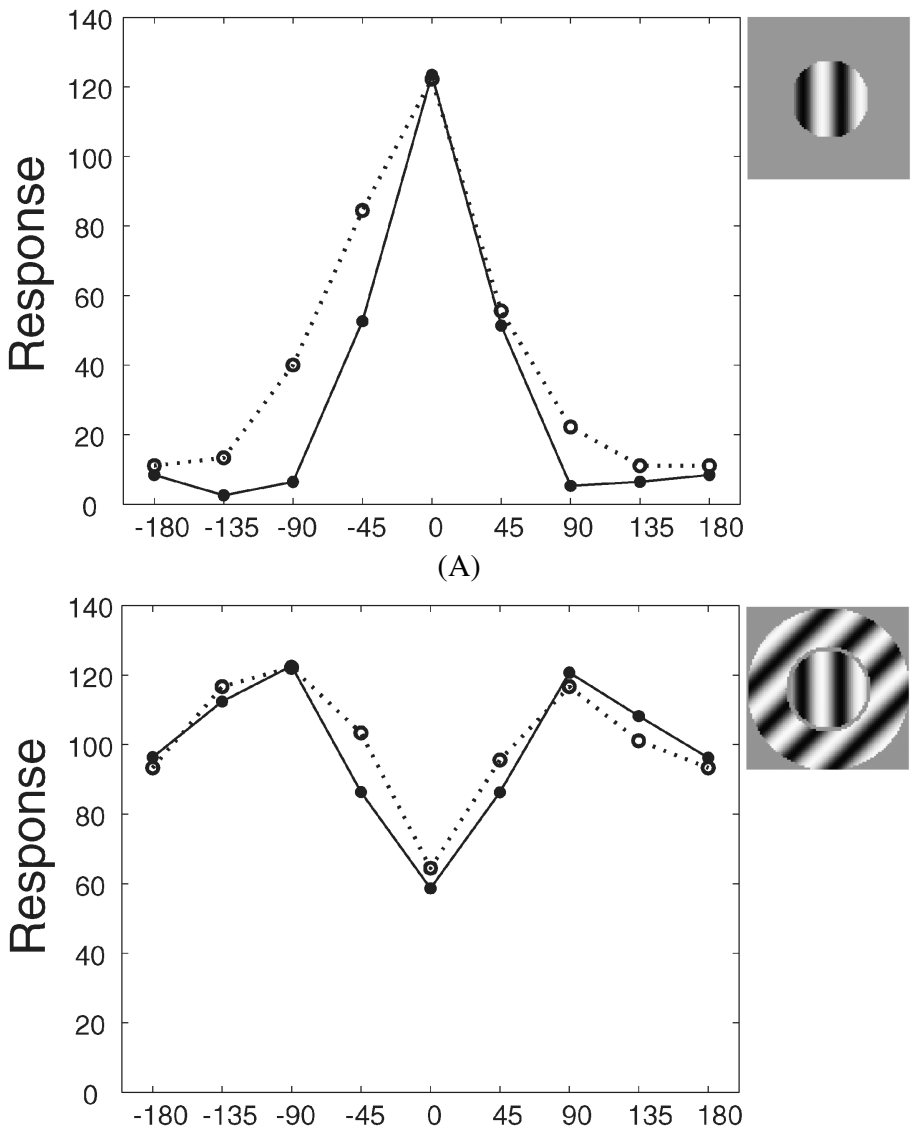

(B)

Figure 2. Tuning to relative direction in the generalized energy model. (A) The center of a directionally selective model complex cell is stimulated with a drifting grating. The size of the grating is confined to a circle that covers the CRF of the model cell (inset). The diameter of the inner circle is $d_{i}=2 \mathrm{deg}$. The direction of motion of the grating is varied from -180 to $180 \mathrm{deg}$ in steps of $45 \mathrm{deg}$. The mean response is plotted as a function of the angle of motion (black dots). The model complex cell gives its best response for gratings moving at an angle of zero degrees and responds only weakly to gratings which move in the opposite direction. For comparison, the tuning curve of a real complex cell from monkey V1 is plotted together with the simulation data (open circles) (Cavanaugh $e t$ al., 2002). The model results are scaled to fit the experimental values which are plotted as presented in the original paper. (B) A surround grating confined to a circular annulus which lies outside the center of the CRF is added to the stimulus (inset). The diameter of the outer annulus is $d_{0}=2 \mathrm{deg}$. We compute the mean response of the model cell for a center grating moving in the preferred direction of the model cell as a function of the direction of motion of the surround grating (black dots). The tuning exhibits clear selectivity for relative direction of motion of center and surround, with best responses for orthogonal surround gratings, intermediate responses for surround gratings moving in the opposite direction, and minimal responses for surround gratings that move in the same direction as the center grating. The simulation data gives a good fit to experimentally derived tuning curves from V1 (open circles) (Cavanaugh et al., 2002). (C) When the center grating is moving in a suboptimal direction (45 deg) (inset), the tuning curve to relative direction flattens, a phenomenon that is also seen in real V1 cells (open circles) (Cavanaugh et al., 2002). 


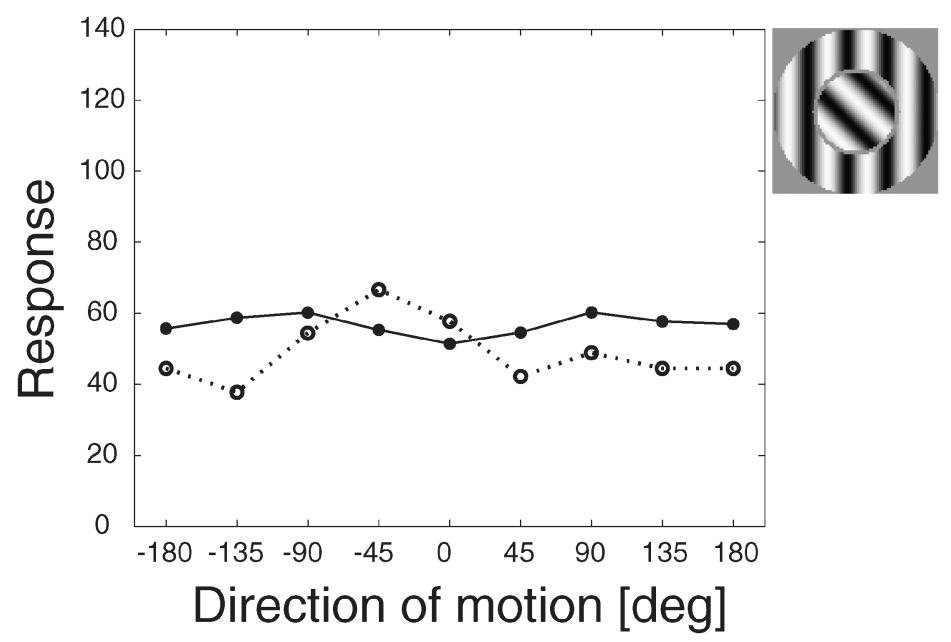

(C)

Figure 2. (Continued.)

We further compute the model complex cell's response for different choices of the spatial frequency of the center stimulus. Results are obtained for surround stimulus orientations of 90, 45 and 0 deg, plotted in Fig. 3B as dashed black, solid black, and gray solid lines, respectively. The model complex cell's sensitivity to orientation contrast depends on the spatial frequency of the center stimulus. This model prediction could be tested by presenting the same stimulus to real V1 neurons.

Influence of orientation selectivity on orientation-contrast sensitivity. In further simulations, we have examined the extent to which the cell's selectivity for orientation determines its sensitivity to orientation contrast. The 'orientation selectivity index' is defined as the ratio of the mean response to a moving center grating of least effective orientation to the mean response to a center grating that moves in the preferred direction of the cell, in the absence of stimulation of the surround. To change the orientation selectivity of the model complex cell, we include orientations with $w^{1}=w^{7}=0.15$ and $w^{2}=w^{6}=0.12$ in the central spatiotemporal filter. The weight of the spatial component with $j=0$ is varied from 0.5 to 10 , resulting in different values for the orientation selectivity index. The sensitivity to orientation contrast is quantified by the 'orientation modulation index', which is the ratio of the mean response to a stimulus configuration of high orientation contrast to the mean response to a stimulus configuration of zero or low orientation contrast. For the high orientation-contrast stimulus, we use a surround grating in motion orthogonal to a center grating (Fig. 4A, inset). For the zero orientation-contrast stimulus, we use a surround grating that moves in the same direction as the center grating. The center grating moves in the preferred direction of the cell in both cases. In Fig. 4A, the orientation modulation index computed for four model cells and is plotted as a function of the orientation selectivity index 


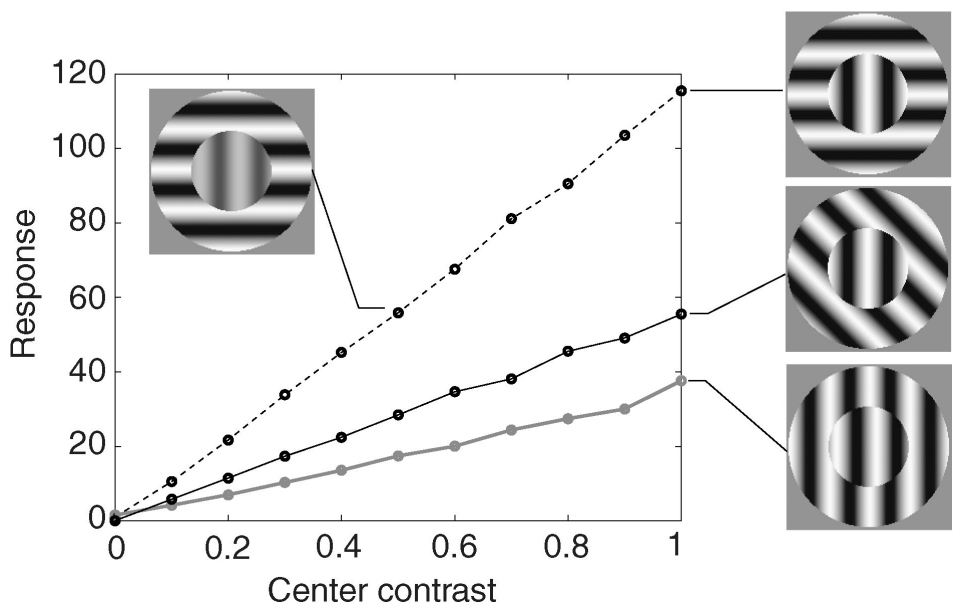

(A)

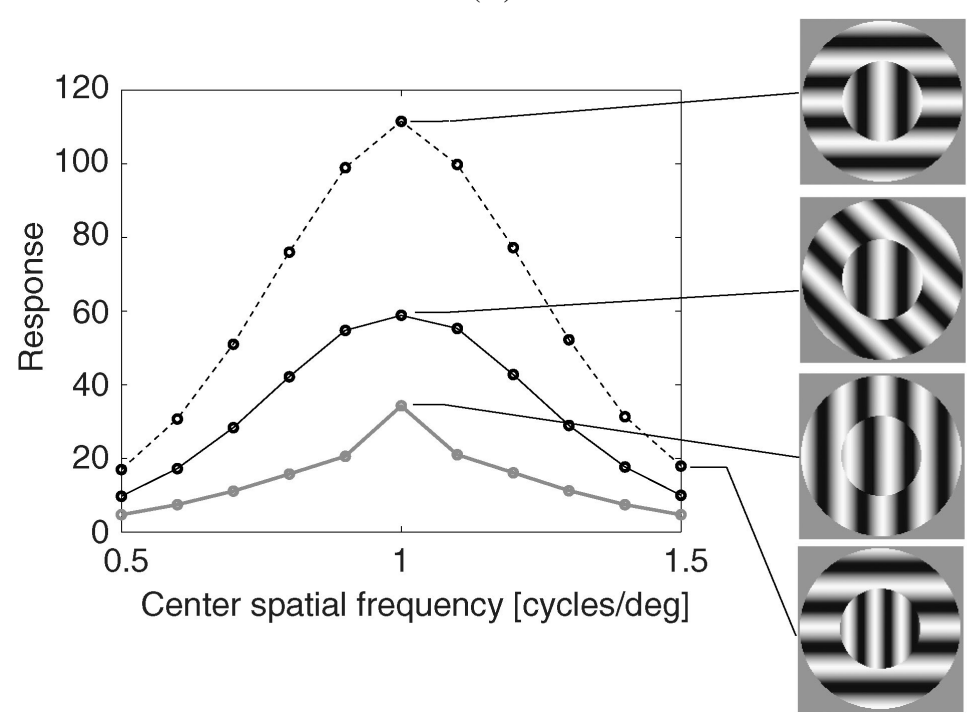

(B)

Figure 3. Influence of center-stimulus attributes on orientation-contrast selectivity. (A) We compute the responses of directionally-selective model complex cells as a function of the center stimulus contrast for surround-stimulus orientations of 90,45 , and $0 \mathrm{deg}$, plotted as dashed black, solid black, and solid gray lines, respectively. The model complex cell has a preferred orientation of $0 \mathrm{deg}$ and a preferred spatial frequency of 1 cycle/ deg. The strength of the surround modulation is dependent on the contrast of the center stimulus. Model parameters are the same as in Fig. 2. (B) We compute the response of the same model neuron as a function of the center stimulus spatial frequency for surroundstimulus orientations of 90,45 and 0 deg, plotted as dashed black, solid black, and solid gray lines, respectively. The strength of the surround modulation is dependent on the spatial frequency of the center stimulus.

(black dots) for the particular stimulus type. The dots corresponding to a particular model-cell type, characterized by the model parameters that are not being varied, are connected with line segments. 
Next, we compute the orientation modulation index with respect to a different orientation-contrast stimulus for the same sample of model cells. The visual scene now consists of a stationary square-shaped window of size $2 \times 2 \mathrm{deg}^{2}$ which contains a moving pattern of oriented stripes (Fig. 4B, inset). The square window is surrounded by a moving pattern of stripes having either orthogonal orientation (high orientation-contrast configuration) or the same orientation (zero orientation-contrast configuration). The size of the background is $12 \times 12 \mathrm{deg}$. The pattern in the center and that in the surround move with the same velocity and in the same direction. The modulation index for orientation is plotted as a function of the orientation-selectivity index (Fig. 4B, black dots).

The results for both stimulus types show that the model complex cell's sensitivity to orientation contrast is independent of its selectivity to orientation, a result that seems to be in agreement with experimental observations.

Influence of directional selectivity on motion-contrast sensitivity. We have also studied the sensitivity of the model to motion contrast as a function of the cell's directional selectivity. The 'direction selectivity index' is the ratio of the response to a center grating moving in the least preferred direction of motion to the response to a center grating moving in the preferred direction of motion. The direction selectivity of the model complex cell is varied by changing the phase $\phi$ of the temporal filter $g(t)$. The sensitivity to motion contrast is quantified by the 'direction modulation index', which is the ratio of the mean response to a stimulus configuration of high motion contrast to the mean response to a stimulus configuration of zero or low motion contrast. For the high motion-contrast stimulus, we use a surround grating moving in the direction opposite to that of a center grating. For the zero motioncontrast stimulus, we use surround grating that moves in the same direction as the center grating (Fig. 5A, inset). The center stimulus moves in the preferred direction of the cell. The direction modulation index is computed for four model cells and plotted as a function of the directional-selectivity index (Fig. 5A, black dots). The dots corresponding to a particular model-cell type, characterized by the model parameters that are not being varied, are connected with line segments.

Next, we compute the direction modulation index of the same sample of model complex cells for a different stimulus type. The visual stimulus consists of a square window of size $2 \times 2 \mathrm{deg}^{2}$ that contains a moving random-dot pattern (Fig. 5B, inset). The window is surrounded by a moving random-dot background that moves in the direction opposite to the pattern in the high-feature-contrast condition, and in the same direction in the low-feature-contrast condition. The size of the background is $12 \times 12 \mathrm{deg}$. Experimentally derived direction modulation indices from V1 are plotted for comparison (Fig. 5B, open circles) (Lamme, 1995).

The results for both stimulus types demonstrate that the model complex cell's sensitivity to motion contrast is independent of its directional selectivity, which is in agreement with experimental observations. 


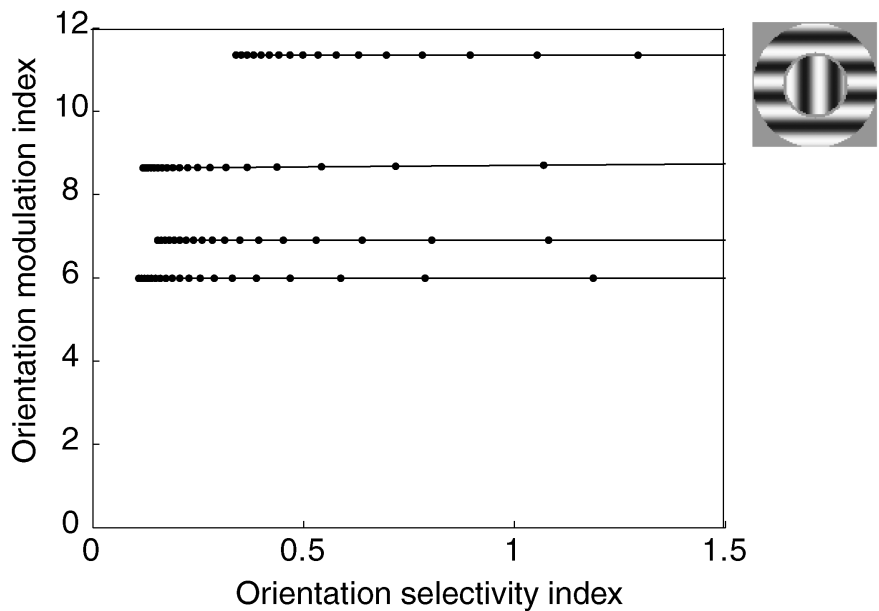

(A)
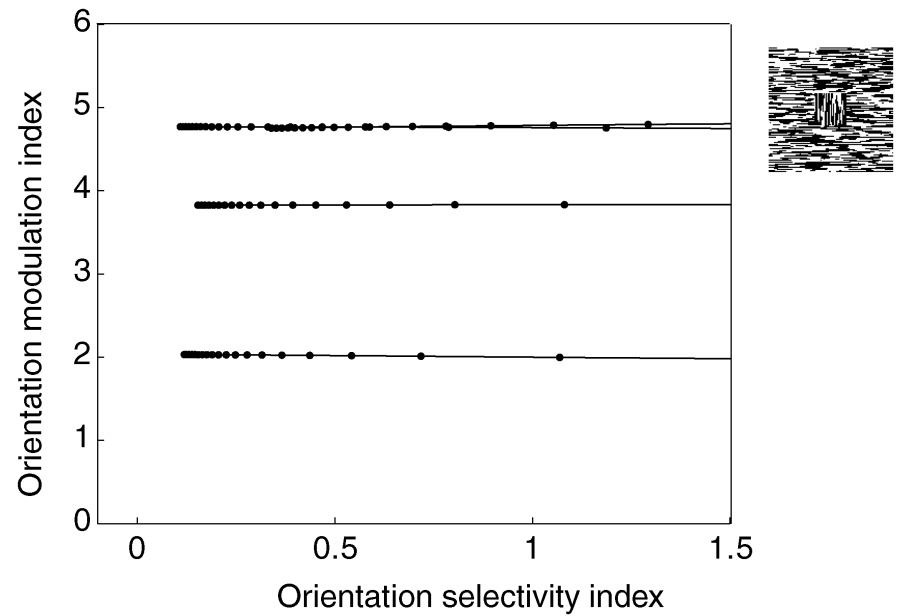

(B)

Figure 4. Orientation-contrast sensitivity as a function of orientation selectivity. (A) We compute the orientation modulation index for a grating stimulus (inset) at different orientation-selectivity indices (see text). The orientation modulation index is plotted as a function of the orientation-selectivity index of the model cell (black dots). No dependence of the modulation index on orientation selectivity is observed. The dots corresponding to a particular model-cell type, characterized by the model parameters that are not being varied, are connected with line segments. (B) We compute the orientation modulation index for an orientation-contrast stimulus (inset) at different orientation-selectivity indices (see text). The visual stimulus consists of a square-shaped window of size $2 \times 2 \mathrm{deg}^{2}$ that contains a moving striped pattern oriented parallel to the $y$-axis. The window is surrounded by a moving striped background of perpendicular orientation in the high orientation-contrast condition, and parallel in the low-contrast condition. The stripes in the window and the surround are moving with the same velocity diagonal downwards (inset). The orientation modulation index is plotted as a function of the orientation-selectivity index of the model cell (black dots). No dependence of the orientation modulation index on orientation selectivity is observed. The dots corresponding to a particular modelcell type, characterized by the model parameters that are not being varied, are connected with line segments. 


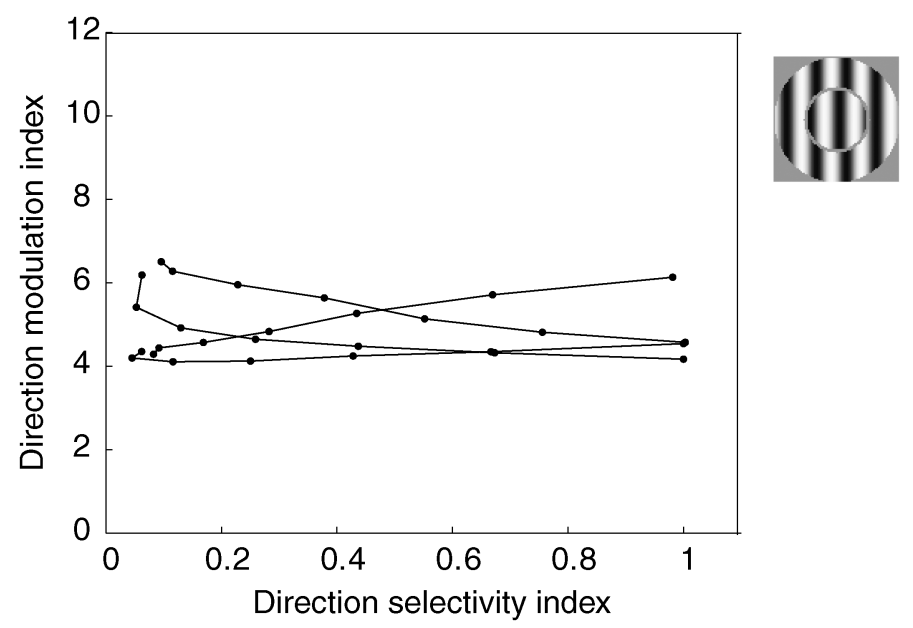

(A)
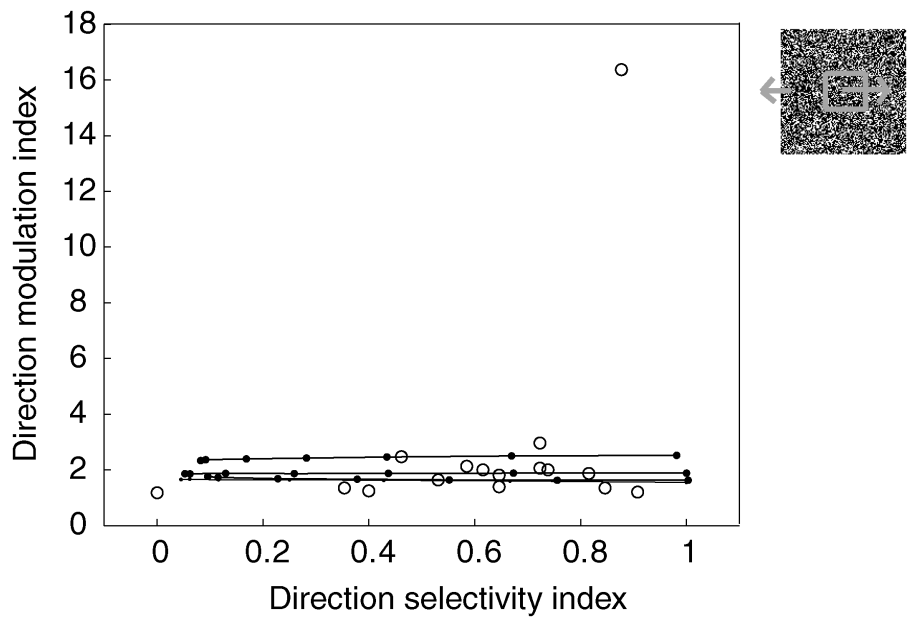

(B)

Figure 5. Motion-contrast sensitivity as a function of direction selectivity. (A) We compute the direction modulation index for a grating stimulus (inset) at different direction-selectivity indices (see text). The direction modulation index is plotted as a function of the direction-selectivity index of the model cell (black dots). No significant dependence of the direction modulation index on direction selectivity is observed. The dots corresponding to a particular model-cell type, characterized by the model parameters that are not being varied, are connected with line segments. (B) We compute the direction modulation index for a motion-contrast stimulus (inset) at different direction-selectivity indices (see text). The visual stimulus consists of a square-shaped window of size $2 \times 2 \mathrm{deg}^{2}$ that contains a moving random-dot pattern. The window is surrounded by a moving random-dot background which moves in the opposite direction in the high direction-contrast condition, and in the same direction in the low-contrast condition. The direction modulation index is plotted as a function of the direction selectivity index of the model cell (black dots). No dependence of the direction modulation index on directional selectivity is observed. The results are in agreement with experimental data from primary visual cortex obtained for the same type of stimulus (open circles) (Lamme, 1995). The dots corresponding to a particular model-cell type, characterized by the model parameters that are not being varied, are connected with line segments. 
Orientation pop out. To illustrate the functional implications of orientationcontrast sensitivity, we have investigated the response of a population of model complex cells to orientation-contrast-defined figures. The visual scene consists of a stationary square window $2 \times 2 \mathrm{deg}^{2}$ in size which contains a moving pattern of oriented stripes (Fig. 6A, inset). The square window is surrounded by a moving pattern of stripes of opposite orientation in the high orientation-contrast configuration (Fig. 6A, inset). The size of the background is $22 \times 22 \mathrm{deg}^{2}$ which is large enough to avoid edge effects in the computation. The pattern in the center and in the surround move with the same speed and in the same direction. In Fig. 6A, the mean population orientation modulation index of a linear array of 20 model complex cells with zero mean population orientation selectivity index is plotted as a function of the position of the CRF center $\mathbf{x}_{0}$ (black dots). The mean response of model complex cells with RF centers located inside the figure are enhanced compared to the response of cells lying outside of the figure. In the population response, the orientation-defined square 'pops out' with sharp borders. Model cell responses for all cells with RF centers located near the edges and near the center of the figure are both enhanced. Experimental data from V1 cells are plotted for comparison (Fig. 6A, open circles) (Lamme, 1995). Compared to an earlier model in which the edges of the figure are usually enhanced compared to the figure center (Dellen et al., 2005), the model results are now in better agreement with the experimental data.

Motion pop out. To illustrate the functional implications of relative-motion sensitivity, we have investigated the response of a population of model complex cells to motion-contrast-defined figures. The visual stimulus consists of a square window of size $2 \times 2 \mathrm{deg}^{2}$ which contains a moving random-dot pattern. The window is surrounded by a moving random-dot background moving in the opposite direction in the high-contrast configuration, and in the same direction in the low-contrast configuration (Fig. 6B, inset). The size of the background is $22 \times 22 \mathrm{deg}^{2}$. In Fig. 6B, the mean population direction modulation index of an array of 20 model complex cells with zero mean population direction selectivity index is plotted as a function of the position of the CRF center $\mathbf{x}_{0}$ (black dots). The mean response of model complex cells with RF centers located inside the figure is enhanced compared to the mean response of model complex cells with RF centers located outside the figure. In the population response, the direction-defined square pops out with sharp borders. Both the edges and the center of the figure are enhanced, in contrast to an earlier model (Dellen et al., 2005). Experimental data from monkey V1 are plotted together with the simulation data (Fig. 6B, open circles) (Lamme, 1995).

\section{Biologically inspired image-segmentation algorithm}

The basic mechanism underlying feature-contrast sensitivity in the generalized energy model of complex cells can be utilized to segment motion-contrast-defined objects from a moving background. A patch of a 2D image is created by setting all the image points located outside the patch equal to zeros. We compute the 

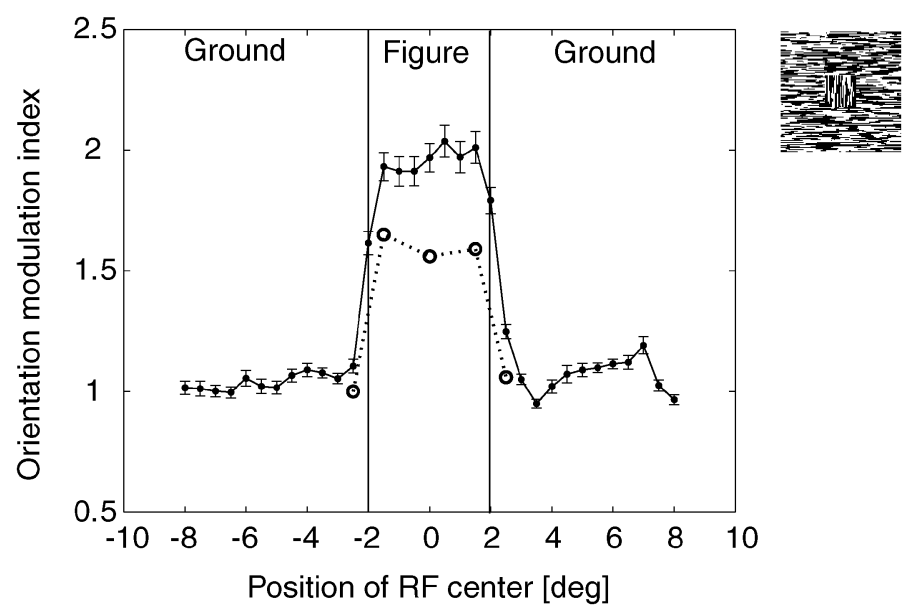

(A)

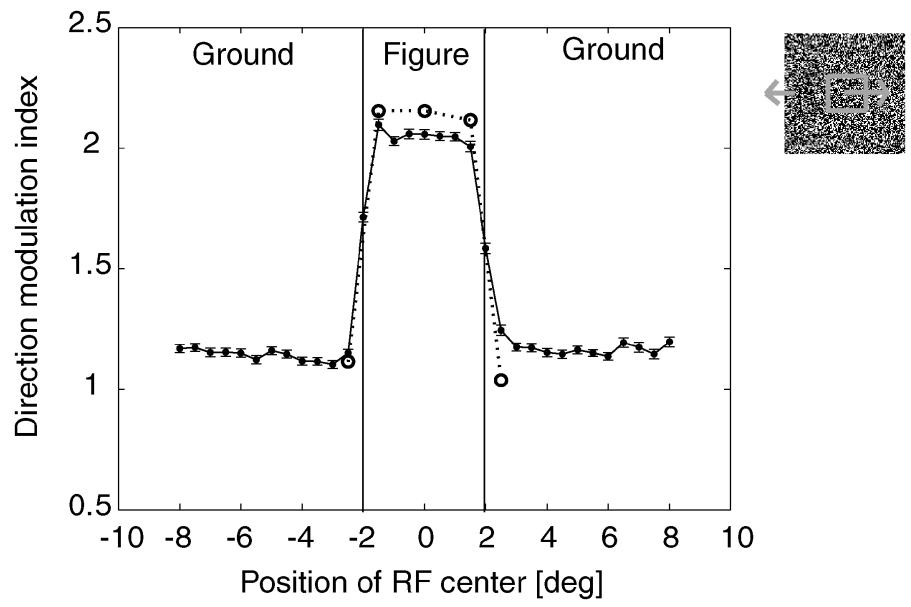

(B)

Figure 6. Computing structure from feature contrast with generalized complex cells. (A) Orientationcontrast induced pop out. The visual stimulus consists of a square-shaped window of size $2 \times 2 \mathrm{deg}^{2}$ that contains a moving striped pattern oriented parallel to the $y$-axis. The window is surrounded by a moving striped background of perpendicular orientation. The stripes in the window and the surround are moving with the same velocity diagonal downwards (inset). The mean orientation modulation index of a population of 20 generalized complex cells with a mean orientation-selectivity index of zero is computed as a function of the location of the center of the model complex cell (black dots). The orientation-contrast-defined square pops out with sharp borders. Both the center as well as the edges are enhanced. The result is in agreement with pop-out effects that have been observed in monkey V1 for the same stimulus (Lamme, 1995) (open circles). (B) Motion-contrast induced pop out. The visual stimulus consists of a square-shaped window of size $2 \times 2 \mathrm{deg}^{2}$ that contains a moving randomdot pattern. The window is surrounded by a moving random-dot background moving in the opposite direction. The mean direction modulation index of a population of 20 generalized complex cells with a mean directional-selectivity index of zero is computed as a function of the location of the center of the model complex cell (black dots). The motion-contrast-defined square pops out with sharp borders. Both the center as well as the edges are enhanced. The result is in agreement with pop-out effects that have been observed in monkey V1 for the same stimulus (Lamme, 1995) (open circles). 
Fourier transform of the patch and multiply every point of Fourier space with the equivalent complex conjugate point of the Fourier transform of the image. The components of the non-linearly transformed images are summed. This procedure is applied to every image of the image sequence. Finally, we differentiate the timedependent output and take its absolute value. We repeat the algorithm for other spatial locations of the local patch to cover the whole image. The algorithm is demonstrated for a moving opaque T-shaped random-dot object that moves in front of a random-dot background. The T-shaped random-dot object moves to the right while the background moves to the left. The object and the background are moving with the same absolute speed of one pixel/time-step. A schematic of the stimulus is presented in Fig. 7A. A mask in the shape of the object is overlayed on the snapshot of the input stimulus for illustrative purposes only. In the algorithm, the T-shaped object is only defined by its motion relative to the background. No brightness cues are given. In Fig. 7B, a snapshot of the output is shown. The T-shaped figure is clearly distinguished from the background. It matches the shape of the original object.

\section{DISCUSSION}

We have proposed a generalization of the standard energy model of complex cells (Adelson and Bergen, 1985) by including complex spatiotemporal filters from the non-CRF in the model. This gives rise to two types of input, characterizing in turn the CRF and non-CRF areas. In the generalized energy model, interactions between stimuli within the CRF and non-CRF are sensitive to contrast of orientation and motion. In particular, the response of a generalized complex cell to a moving grating covering the CRF is modulated when a moving grating is added to the surround. The strongest responses are obtained for gratings moving orthogonal to each other, with intermediate responses for gratings moving in opposite directions and minimum responses for gratings moving in the same direction. The response to orientation contrast is dependent on the contrast and spatial frequency of the center stimulus. In the model, orientation-contrast sensitivity decreases with decreasing contrast of the center stimulus. Further, if the spatial frequency of the center stimulus deviates from the preferred spatial frequency of the model neuron, orientation-contrast sensitivity is reduced. The model's orientation- and direction-contrast sensitivities are independent of the model cell's selectivity for orientation and direction. The results are in agreement with experimental data from monkey primary visual cortex (Cavanaugh et al., 2002; Kastner et al., 1999; Lamme, 1995; Levitt and Lund, 1997; Sillito et al., 1995). The generalized energy model proposed in this paper is a functional description of cortical cells. It does not attempt to model the precise biophysical mechanisms underlying cortical functions.

The sensitivity of the model to feature contrast between a center stimulus and a surround stimulus has important functional implications for the processing of figures whose boundaries are defined by a stimulus context. In the model, pop-out 


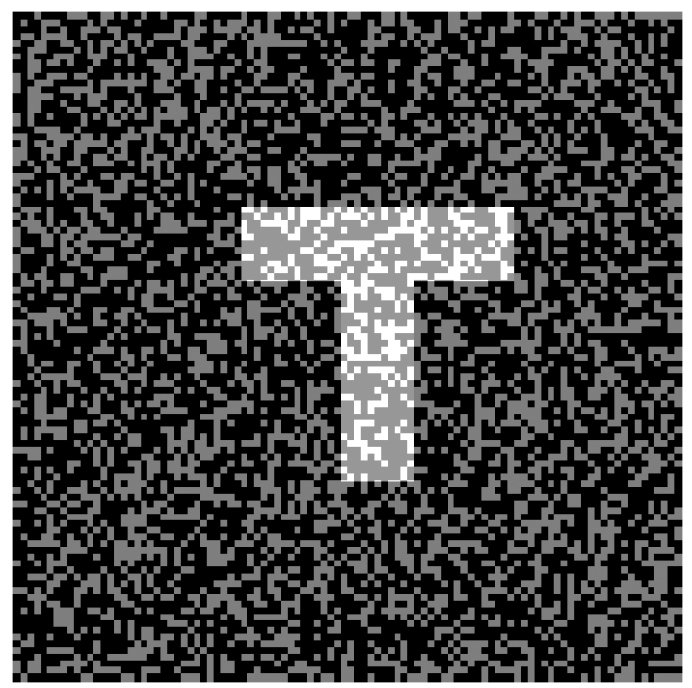

(A)

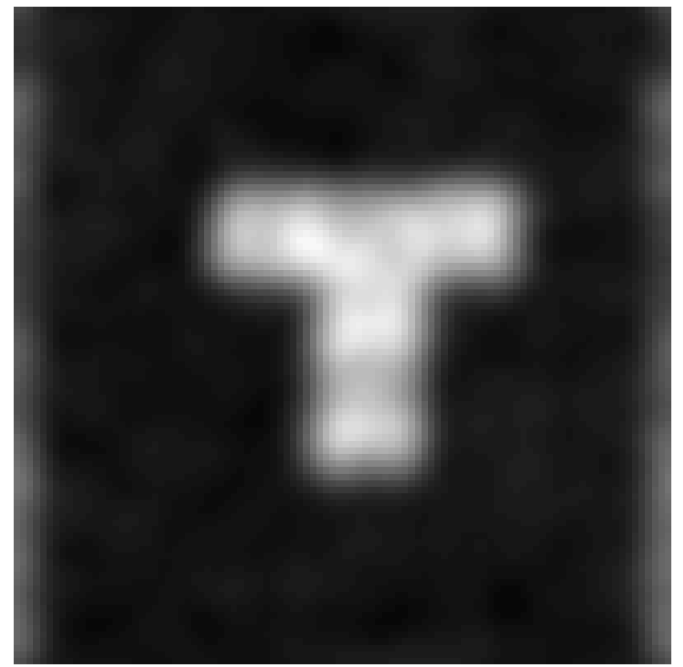

(B)

Figure 7. Biologically inspired algorithm for the computation of structure from motion contrast. (A) A T-shaped random-dot object is moving in front of a random-dot background of equal density and intensity. The T-shaped object is only defined by its motion relative to the background. The background is moving to the left while the object moves to the right. No other cues are given. A snapshot of the image sequence is presented. For illustrative purposes, the T-shaped object is highlighted. (B) We compute the Fourier transform of a local spatial patch of each 2D image of the sequence and multiply every component with the complex conjugate of the Fourier transform of the image. The components of the non-linearly transformed images are summed. This procedure is applied to every image of the image sequence. Finally, we differentiate the time-dependent output and take its absolute value. We repeat the algorithm for other spatial locations of the local patch to cover the whole image. A snapshot of the output sequence is presented. The T-shaped objects pops out from the background with sharp borders. The motion-defined object can thus be tracked continuously from frame to frame. 
effects due to orientation and motion contrast are observed that closely resemble pop-out phenomena in measured V1 responses (Lamme, 1995).

The mechanism underlying feature-contrast sensitivity in the generalized model was further used to develop a biologically inspired algorithm for the computation of structure from motion, which allows motion-defined objects to be detected and tracked continuously from frame to frame. The algorithm might be useful for machine vision applications.

Previous models of contextual modulation in V1 are mostly based on the assumption that surround cells selectively inhibit cells with similar orientation or direction preference, for example via inhibitory interneurons (Dragoi and Sur, 2000; Grigorescu et al., 2003; Somers et al., 1998; Stemmler et al., 1995; Zhaoping, 2003). In the model of Dragoi and Sur (2000), facilatory effects are due to disinhibition caused by a second population of inhibitory neurons. These models have been successful in describing contextual effects of V1 neurons. However, some properties of contextual effects remain elusive. For example, the orientation-contrast and motion-contrast sensitivities have been shown to be independent of the orientation/direction selectivity index of the neuron (Lamme, 1995). Even fully unselective cells show highly significant figure-ground enhancement in response to orientationcontrast- and motion-contrast-defined figures. These phenomena are not predicted by models employing simple local rules such as binding by similarity in feature space. In the generalized energy model of complex cells, both orientation-contrast and direction-contrast sensitivity are largely independent of the selectivity for orientation and motion direction inherent to the complex cell. The contextual effects observed in the model originate from nonlinear interactions of spatiotemporal filters. In contrast, mechanisms for feature-contrast detection based on local rules, for example inhibitory effects between cells with similiar orientation and direction selecitivities, are expected to be highly dependent on the selectivity of the neuron for the particular feature. In particular, a neuron which responds to motion in both the left and the right direction will not become sensitive to motion contrast by introducing inhibition from surround neurons of similar direction preference. Until now, this argument has served as a support for feedback models. The computation of feature contrast through energy computations proposed here provides an alternative explanation which does not require feedback.

Orientation contrast sensitivity is related to endstopping, i.e. the selectivity for bars of a specific length (Hubel and Wiesel, 1962). A mathematical model of enstopped neurons due to Dobbins et al. (1987) showed that endstopping provides a basis for the calculation of curvature, which can be understood as orientation contrast along a line. In their model, the response of a simple cell is inhibited by another simple cell having a larger receptive field. The contributions of the two cells are combined via a subtraction followed by a rectification. In our model, we also combine responses of model simple cells with receptive fields spanning different areas of the visual field, but the model simple cell responses are combined using multiplication, giving rise to nonlinear response properties of the model neurons. 
The proposed model can in principle be implemented by a combination of feedforward and recurrent connections. In this picture, feedforward input either from simple cells or from the LGN creates the CRF, or, in terms of our mathematical formulation, the mathematically complex linear spatiotemporal filter $s(t)$. This filter defines the feature selectivity of the complex cells. Recurrent connections draw in linear filters form a large area of the visual space and are combined nonlinearly with the feedforward input, maybe at the synaptic stage. This scenario agrees with experimental data, which suggest that simple cells behave more like complex cells when the effect of recurrent excitatory connections is enhanced (Sillito et al., 1995). Further support comes from recent findings that complex cells are best described using more than one pair of linear filters (Rust et al., 2005). Furthermore, the generalized energy model contains a normalization which could be implemented by a uniform surround inhibition or a gain control mechanism.

In conclusion, we find that the nonlinear transformations proposed in the generalized energy model serve as a feature-contrast detector and reproduce measured V1 tuning curves. These nonlinear transformations also produce phase independence a property which distinguishes complex cells from simple cells. In the future, it could be clarified how far energy computations play a functional role for the computation of feature contrast in V1 by measuring whether feature-contrast sensitivity and phase independence are correlated.

\section{Acknowledgements}

The work was supported by a grant from the NIH RO1 EY15678 to R. W. B. K. D. acknowledges financial support by the German Ministry for Education and Research (BMBF) via the Bernstein Center for Computational Neuroscience (BCCN) Göttingen under Grant No. 01GQ0430.

\section{REFERENCES}

Adelson, E. H. and Bergen, J. R. (1985). Spatiotemporal energy models for the perception of motion, J. Opt. Soc. Am. A 2, 284-299.

Albright, T. D. and Stoner, G. R. (2002). Contextual influences on visual processing, Annu. Rev. Neurosci. 25, 339-379.

Cao, A. and Schiller, P. H. (2003). Neural responses to relative speed in the primary visual cortex of rhesus monkey, Visual Neuroscience 20, 77-84.

Cavanaugh, J. R., Wyeth, B. and Movshon, J. A. (2002). Selectivity and spatial distribution of signals from the receptive field surround in macaque V1 neurons, J. Neurophysiol. 88, 2547-2556.

Chance, F. S., Nelson, S. B. and Abbott, L. F. (1999). Complex cells as cortically amplified simple cells, Nature Neuroscience 2, 277-282.

Chen, Y., Wang, Y. and Qian, N. (2001). Modeling V1 disparity tuning to time-varying stimuli, J. Neurophysiol. 86, 143-155.

DeAngelis, G. C., Ohzawa, I. and Freeman, R. D. (1993). Spatiotemporal organization of simple-cell receptive fields in the cat's striate cortex. I. General characteristics and postnatal development, J. Neurophysiol. 69, 1091-1117. 
Dellen, B. K., Wessel, R. and Clark, J. W. (2005). Computing relative motion with complex cells, Visual Neuroscience 22, 225-236.

Dobbins, A., Zucker, S. W. and Cynader, M. S. (1987). Endstopped neurons in the visual cortex as a substrate for calculating curvature, Nature 329, 438-441.

Dragoi, V. and Sur, M. (2000). Dynamic properties of recurrent inhibition in primary visual cortex: Contrast and orientation dependence of contextual effects, J. Neurophysiol. 83, 1019-1030.

Emerson, R. C., Bergen, J. R. and Adelson, E. H. (1992). Directionally selective complex cells and the computation of motion energy in cat visual cortex, Vision Research 32, 201-218.

Grigorescu, C., Petkov, N. and Westenberg, M. A. (2003). Contour detection based on nonclassical receptive field inhibition, IEEE Transactions on Image Processing 12, 729-739.

Grosof, D. H., Shapley, R. M. and Hawken, M. J. (1993). Macaque V1 neurons can signal 'illusory' contours, Nature 365, 550-552.

Hegde, J. and Felleman, D. J. (2003). How selective are V1 cells for pop-out stimuli?, J. Neurosci. 23, 9968-9980.

Hubel, D. H. and Wiesel, T. N. (1962). Receptive fields, binocular interaction and functional architecture in the cat's visual cortex, J. Physiol. (Lond.) 160, 106-154.

Jones, H. E., Grieve, K. L., Wang, W. and Sillito, A. M. (2001). Surround suppression in primate V1, J. Neurophysiol. 86, 2011-2028.

Kapadia, M. K., Ito, M., Gilbert, C. D. and Westheimer, G. (1995). Improvement in visual sensitivity by changes in local context: parallel studies in human observers and in V1 of alert monkeys, Neuron 15, 843-856.

Kastner, S., Nothdurft, H.-C. and Pigarev, I. N. (1997). Neuronal correlates of pop-out in cat striate cortex, Vision Research 37, 371-376.

Kastner, S., Nothdurft, H. C. and Pigarev, I. N. (1999). Neuronal responses to orientation and motion contrast in cat striate cortex, Vis. Neurosci. 16, 587-600.

Knierim, J. J. and van Essen, D. C. (1992). Neuronal responses to static texture patterns in area V1 of the alert macaque monkey, J. Neurophysiol. 67, 961-980.

Lamme, V. A. F. (1995). The neurophysiology of figure-ground segregation in primary visual cortex, J. Neurosci. 15, 1605-1615.

Lee, T. S., Mumford, D., Romero, R. and Lamme, V. A. F. (1998). The role of primary visual cortex in higher level vision, Vision Research 38, 2429-2454.

Levitt, J. B. and Lund, J. S. (1997). Contrast dependence of contextual effects in primate visual cortex, Nature 387, 73-76.

Li, C. Y., Lei, J. J. and Yao, H. S. (1999). Shift in speed selectivity of visual cortical neurons: A neural basis of perceived motion contrast, Proc. Natl. Acad. Sci. USA 96, 4052-4056.

MacEvoy, S. P., Kim, W. and Paradiso, M. A. (1998). Integration of surface information in primary visual cortex, Nature Neuroscience 1, 616-620.

Movshon, J., Thompson, I. and Tolhurst, D. (1978a). Spatial summation in the receptive fields of simple cells in the cat's striate cortex, J. Physiol. 283, 53-77.

Movshon, J., Thompson, I. and Tolhurst, D. (1978b). Receptive field organization of complex cells in the cat's striate cortex, J. Physiol. 283, 79-99.

Movshon, J. A., Thompson, I. D. and Tolhurst, D. J. (1978c). Spatial and temporal contrast sensitivity of neurones in areas 17 and 18 of the cat's visual cortex, J. Physiol. 283, 101-120.

Müller, J. R., Metha, A. B., Krauskopf, J. and Lennie, P. (2003). Local signals from beyond the receptive fields of striate cortical neurons, J. Neurophysiol. 90, 822-831.

Orban, G. A., Gulyas, B. and Vogels, R. (1987). Influence of a moving textured background on direction selectivity of cat striate neurons, J. Neurophysiol. 57, 1792-1812.

Qian, N. and Mikaelian, S. (2000). Relationship between phase and energy methods for disparity computation, Neural Computation 12, 279-292.

Ringach, D. L. (2002). Spatial structure and symmetry of simple-cell receptive fields in macaque primary visual cortex, J. Neurophysiol. 88, 455-463. 
Ringach, D. L. (2004). Mapping receptive fields in primary visual cortex, J. Physiol. 558.3, 717-728.

Rossi, A. F., Rittenhouse, C. D. and Paradiso, M. A. (1996). The representation of brightness in primary visual cortex, Science 273, 1055-1056.

Rossi, A. F., Desimone, R. and Ungerleider, L. (2001). Contextual modulation in primary visual cortex of macaques, J. Neuroscience 21, 1698-1709.

Rust, N. C., Schwartz, O., Movshon, J. A. and Simoncelli, E. P. (2005). Spatiotemporal elements of macaque V1 receptive fields, Neuron 46, 945-956.

Sillito, A. M., Grieve, K. L., Jones, H. E., Cudeiro, J. and Davis, J. (1995). Visual cortical mechanisms detecting focal orientation discontinuities, Nature 378, 492-496.

Skottun, B. C., De Valois, R. L., Grosof, D. H., Movshon, J. A., Albrecht, D. G. and Bonds, A. B. (1991). Classifying simple and complex cells on the basis of response modulation, Vision Research 31, 1079-1086.

Somers, D. C., Todorov, E., Siapas, A. G., Toth, L. J., Kim, D. and Sur, M. (1998). A local circuit approach to understanding integration of long-range inputs in primary visual cortex, Cereb. Cortex 8, 204-217.

Stemmler, M., Usher, M. and Niebur, E. (1995). Lateral interactions in primary visual cortex: a model bridging physiology and psychophysics, Science 269, 1877-1880.

Touryan, J., Felsen, G. and Dan, Y. (2005). Spatial structure of complex receptive fields measured with natural images, Neuron 45, 781-791.

Walker, G. A., Ohzawa, I. and Freeman, R. D. (2000). Suppression outside the classical receptive field, Visual Neuroscience 17, 369-379.

Zhaoping, L. (2003). V1 mechanisms and some figure-ground and border effects. J. Physiol. 97, 503515.

Zipser, K., Lamme, V. A. and Schiller, P. H. (1996). Contextual modulation in primary visual cortex, J. Neurosci. 16, 7376-7389. 